\title{
A Study of Doubly Warped Product Immersions in a Nearly Trans-Sasakian Manifold with Slant Factor
}

\author{
Ali H. Alkhaldi, ${ }^{1}$ Aliya Naaz Siddiqui $\mathbb{D}^{2}$, Kamran Ahmad, $^{2}$ and Akram Ali $\mathbb{D}^{1}$ \\ ${ }^{1}$ Department of Mathematics, College of Science, King Khalid University, 9004 Abha, Saudi Arabia \\ ${ }^{2}$ M.M. Engineering College, Maharishi Markandeshwar (Deemed to be University), Mullana, Ambala 133207, India \\ Correspondence should be addressed to Akram Ali; akramali133@gmail.com
}

Received 10 July 2021; Accepted 14 September 2021; Published 4 October 2021

Academic Editor: Zine El Abiddine Fellah

Copyright (c) 2021 Ali H. Alkhaldi et al. This is an open access article distributed under the Creative Commons Attribution License, which permits unrestricted use, distribution, and reproduction in any medium, provided the original work is properly cited.

\begin{abstract}
In this article, we discuss the de Rham cohomology class for bislant submanifolds in nearly trans-Sasakian manifolds. Moreover, we give a classification of warped product bislant submanifolds in nearly trans-Sasakian manifolds with some nontrivial examples in the support. Next, it is of great interest to prove that there does not exist any doubly warped product bislant submanifolds other than warped product bislant submanifolds in nearly trans-Sasakian manifolds. Some immediate consequences are also obtained.
\end{abstract}

\section{Introduction and Motivations}

The most inventive topic in the field of differential geometry currently is the theory of warped product manifolds. These manifolds are the most fruitful and natural generalization of Riemannian product manifolds. Due to the important roles of the warped product in mathematical physics and geometry, it has become the most active and interesting topic for researchers, and many nice results are available in the literature (see [1-3]).

Chen $[4,5]$ initiates the concept of warped product submanifolds by proving the nonexistence result of warped product CR-submanifolds of type $\mathcal{N}_{\perp} \times{ }_{f} \mathcal{N}_{T}$ in Kähler manifolds, where $\mathcal{N}_{\perp}$ and $\mathcal{N}_{T}$ are anti-invariant and invariant submanifolds, respectively. Moreover, he considers warped product CR-submanifolds of type $\mathcal{N}_{T} \times_{f} \mathcal{N}_{\perp}$ and gives an inequality involving a warping function $f$ and the squared norm of the second fundamental form $\|h\|^{2}$.

On the other hand, the concept of ordinary warped products can be extended to doubly warped products. By using this generalization, Sahin [6] shows that there exist no doubly warped product CR-submanifolds in Kähler manifolds other than warped product CR-submanifolds. He also investigates the existence of doubly twisted product CRsubmanifolds in the same ambient. Many geometers have obtained several results on warped products and doubly warped products [7-12].

The concept of bislant submanifolds is defined by Cabrerizo et al. [13] as the natural generalization of contact CR-, slant, and semislant submanifolds. Such submanifolds generalize invariant, anti-invariant, and pseudoslant submanifolds as well. Recently, the warped product bislant submanifolds in nearly trans-Sasakian manifolds is studied by Siddiqui et al. in [1]. They obtain several inequalities for the squared norm of the second fundamental form in terms of a warping function $f$.

In this paper, firstly, we discuss the de Rham cohomology class for closed bislant submanifolds in a nearly transSasakian manifold. Secondly, in view of embedding theorem of Nash [14], we study an isometric immersion of a warped product bislant submanifold into an arbitrary nearly transSasakian manifold. Then, we investigate the existence of doubly warped products in the same ambient.

\section{Nearly Trans-Sasakian Manifolds and their Submanifolds}

Definition 1 (see [15]). A $(2 m+1)$-dimensional differentiable manifold $\overline{\mathcal{N}}$ is said to have an almost contact structure $(\phi, \xi, \eta, g)$ if there exists on $\overline{\mathcal{N}}$, where 
(i) a tensor field $\phi$ of type $(1,1)$

(ii) a vector field $\xi$

(iii) a 1 -form $\eta$

(iv) a Riemannian metric $g$

such that

$\phi^{2}=-I+\eta \otimes \xi, \phi \xi=0, \eta(\xi)=1, \eta \circ \phi=0, \eta(X)=g(X, \xi)$,

$g(\phi X, \phi Y)=g(X, Y)-\eta(X) \eta(Y), g(\phi X, Y)+g(X, \phi Y)=0$,

for any $X, Y \in T \overline{\mathcal{N}}$.

The covariant derivative of the tensor field $\phi$ is given by

$$
\left(\bar{\nabla}_{X} \phi\right) Y=\bar{\nabla}_{X} \phi Y-\phi \bar{\nabla}_{X} Y
$$

for any $X, Y \in T \overline{\mathcal{N}}$.

In 2000, Gherghe introduced a notion of nearly transSasakian structure of type $(\alpha, \beta)$, which generalizes the trans-Sasakian structure. A nearly trans-Sasakian structure of type $(\alpha, \beta)$ is called nearly $\alpha$-Sasakian (resp. nearly $\beta$-Kenmotsu) if $\beta=0$ (resp. $\alpha=0$ ).

Definition 2 (see [16]). An almost contact metric structure $(\phi, \xi, \eta, g)$ on $\overline{\mathcal{N}}$ is called a nearly trans-Sasakian structure if

$\left(\bar{\nabla}_{X} \phi\right) Y+\left(\bar{\nabla}_{Y} \phi\right) X=\alpha(2 g(X, Y) \xi-\eta(Y) X-\eta(X) Y)-\beta(\eta(Y) \phi X+\eta(X) \phi Y)$,

for any $X, Y \in T \overline{\mathcal{N}}$.

Remark 3.

(i) A nearly trans-Sasakian structure of type $(\alpha, \beta)$ is

(a) nearly Sasakian if $\beta=0, \alpha=1$ [17]

(b) nearly Kenmotsu if $\alpha=0, \beta=1$ [18]

(c) nearly cosymplectic if $\alpha=\beta=0$ [19]

(ii) Remark that every Kenmotsu manifold is a nearly Kenmotsu manifold but the converse is not true. Also, a nearly Kenmotsu manifold is not a Sasakian manifold. On another hand, every nearly Sasakian manifold of dimension greater than five is a Sasakian manifold.

We put $\operatorname{dim} \mathcal{N}=n$ and $\operatorname{dim} \overline{\mathcal{N}}=2 m+1$. The Riemannian metric for $\mathcal{N}$ and $\overline{\mathcal{N}}$ is denoted by the same symbol $g$. Let $T \mathcal{N}$ and $T^{\perp} \mathcal{N}$ denote the Lie algebra of the vector field and set of all normal vector fields on $\mathcal{N}$, respectively. The operator of covariant differentiation with respect to the Levi-Civita connection in $\mathcal{N}$ and $\overline{\mathcal{N}}$ is denoted by $\nabla$ and $\bar{\nabla}$ , respectively. The Gauss and Weingarten formulae are respectively given as [15]

$$
\begin{array}{r}
\bar{\nabla}_{X} Y=\nabla_{X} Y+h(X, Y), \\
\bar{\nabla}_{X} \mathscr{V}=-A_{\mathscr{V}}(X)+\nabla_{X}^{\perp} Y,
\end{array}
$$

for any $X, Y \in T \mathscr{N}$ and $\mathscr{V} \in T^{\perp} \mathcal{N}$. Here, $h$ is the second fundamental form, $A$ is the shape operator, and $\nabla^{\perp}$ is the operator of covariant differentiation with respect to the linear connection induced in the normal bundle $T^{\perp} \mathcal{N}$.

The second fundamental form and the shape operator are related as [15]

$$
g(h(X, Y), \mathscr{V})=g\left(A_{\mathscr{V}}(X), Y\right)
$$

for any $X, Y \in T \mathcal{N}$ and $\mathscr{V} \in T^{\perp} \mathcal{N}$. Here, $g$ denote the induced metric on $\mathcal{N}$ as well as the Riemannian metric on $\overline{\mathcal{N}}$.

Let $x \in \mathcal{N}$ and $\left\{\mathscr{E}_{1}, \cdots, \mathscr{E}_{n}\right\}$ be a local orthonormal frame of $T_{x} \mathcal{N}$ and $\left\{\mathscr{E}_{n+1}, \cdots, \mathscr{E}_{2 m+1}\right\}$ be a local orthonormal frame of $T_{x}^{\perp} \mathcal{N}$. The mean curvature vector $\mathscr{H}$ of a submanifold $\mathscr{N}$ at $x$ is given by [15]

$$
\mathscr{H}=\frac{1}{n} \sum_{i=1}^{n} h\left(\mathscr{E}_{i}, \mathscr{E}_{i}\right) .
$$

A submanifold $\mathcal{N}$ of $\overline{\mathcal{N}}$ is said to be [15]

(i) totally umbilical if $h(X, Y)=g(X, Y) \mathscr{H}$, for any $X$, $Y \in T \mathcal{N}$

(ii) totally geodesic if $h(X, Y)=0$, for any $X, Y \in T \mathcal{N}$

(iii) minimal if $\mathscr{H}=0$, that is, trace $h \equiv 0$

For any $X \in T \mathcal{N}$, we put [15]

$$
\phi X=\mathrm{P} X+\mathrm{F} X
$$

where $\mathrm{P} X=$ tangent $(\phi X)$ and $\mathrm{F} X=\operatorname{normal}(\phi X)$. Then $\mathrm{P}$ is an endomorphism of $T \mathscr{N}$, and $\mathrm{F}$ is the normal bundle valued 1 -form on $T \mathcal{N}$. In the same way, for any $\mathscr{V} \in T^{\perp} \mathcal{N}$, we put [15]

$$
\phi \mathscr{V}=\mathbf{B} \mathscr{V}+\mathscr{C} \mathscr{V},
$$

where $\mathbf{B} \mathscr{V}=\operatorname{tangent}(\phi \mathscr{V})$ and $\mathscr{C} \mathscr{V}=\operatorname{normal}(\phi \mathscr{V})$. It is easy to see that $\mathrm{P}$ and $\mathscr{C}$ are skew-symmetric and

$$
g(\mathrm{~F} X, \mathscr{V})=-g(X, \mathbf{B} \mathscr{V})
$$

for any $X \in T \mathscr{N}$ and $\mathscr{V} \in T^{\perp} \mathcal{N}$.

Definition 4. A submanifold $\mathcal{N}$ of an almost contact metric manifold $\overline{\mathcal{N}}$ is said to be invariant if $F \equiv 0$, that is, $\phi X \in T$ 
$\mathcal{N}$, and anti-invariant if $P \equiv 0$, that is, $\phi X \in T^{\perp} \mathcal{N}$, for any $X \in T \mathcal{N}$.

In contact geometry, Lotta introduced slant immersions as follows [20].

Definition 5. Let $\mathcal{N}$ be a submanifold of an almost contact metric manifold $\overline{\mathcal{N}}$. For each nonzero vector $X \in T_{x} \mathcal{N}-\{$ $\left.\xi_{x}\right\}$ and $x \in \mathcal{N}$, the angle $\theta(p) \in[0, \pi / 2]$ between $\phi X$ and $P$ $X$ is called slant angle of $\mathcal{N}$. If slant angle is constant for each $X \in T_{x} \mathcal{N}-\left\{\xi_{x}\right\}$, then the submanifold is called the slant submanifold.

For slant submanifolds, the following facts are known:

$$
\begin{gathered}
\mathrm{P}^{2}(X)=\cos ^{2} \theta(-X+\eta(X) \xi), \\
g(\mathrm{P} X, \mathrm{P} Y)=\cos ^{2} \theta(g(X, Y)-\eta(Y) \eta(X)), \\
g(\mathrm{~F} X, \mathrm{~F} Y)=\sin ^{2} \theta(g(X, Y)-\eta(Y) \eta(X)),
\end{gathered}
$$

for any $X, Y \in T \mathcal{N}$. Here, $\theta$ is slant angle of $\mathcal{N}$.

Remark 6. If we assume

(i) $\theta=0$, then $\mathcal{N}$ is an invariant submanifold

(ii) $\theta=\pi / 2$, then $\mathcal{N}$ is an anti-invariant submanifold

(iii) $\theta(p) \in(0, \pi / 2)$, then $\mathcal{N}$ is a proper slant submanifold

There are some other important classes of submanifolds which are determined by the behavior of tangent bundle of the submanifold under the action of an almost contact metric structure $\phi$ of $\overline{\mathcal{N}}[1]$ :

(i) A submanifold $\mathcal{N}$ of $\overline{\mathcal{N}}$ is called a contact CR-submanifold of $\overline{\mathcal{N}}$ if there exists a differentiable distribution $D$ on $\mathscr{N}$ whose orthogonal complementary distribution $D^{\perp}$ is anti-invariant

(ii) A submanifold $\mathcal{N}$ of $\overline{\mathcal{N}}$ is called a semislant submanifold of $\overline{\mathcal{N}}$ if there exists a pair of orthogonal distributions $D$ and $D_{\theta}$ such that $D$ is invariant and $D_{\theta}$ is proper slant

(iii) A submanifold $\mathcal{N}$ of $\overline{\mathcal{N}}$ is called pseudoslant submanifold of $\overline{\mathcal{N}}$ if there exists a pair of orthogonal distributions $D^{\perp}$ and $D_{\theta}$ such that $D^{\perp}$ is anti-invariant and $D_{\theta}$ is proper slant

Definition 7 (see [13]). A submanifold $\mathcal{N}$ of an almost contact metric manifold $\overline{\mathscr{M}}$ is said to be a bislant submanifold if there exists a pair of orthogonal distributions $D_{\theta_{1}}$ and $D_{\theta_{2}}$ of
$\mathcal{N}$ such that

$$
T \mathcal{N}=D_{\theta_{1}} \oplus D_{\theta_{2}} \oplus\{\xi\}
$$

(i) $\mathrm{P} D_{\theta_{1}} \perp D_{\theta_{2}}$ and $\mathrm{P} D_{\theta_{2}} \perp D_{\theta_{1}}$

(ii) Each distribution $D_{\theta_{i}}$ is slant with slant angle $\theta_{i}$ for $i=1,2$

Remark 8. If we assume

(i) $\theta_{1}=0$ and $\theta_{2}=\pi / 2$, then $\mathcal{N}$ is a CR-submanifold

(ii) $\theta_{1}=0$ and $\theta_{2} \neq 0, \pi / 2$, then $\mathcal{N}$ is a semislant submanifold

(iii) $\theta_{1}=\pi / 2$ and $\theta_{2} \neq 0, \pi / 2$, then $\mathcal{N}$ is a pseudoslant submanifold

(iv) $\theta_{1}, \theta_{2} \in(0, \pi / 2)$, then $\mathcal{N}$ is a proper bislant submanifold

For a bislant submanifold $\mathcal{N}$ of an almost contact metric manifold, the normal bundle of $\mathcal{N}$ is decomposed as

$$
T^{\perp} \mathcal{N}=\mathrm{F} D_{\theta_{1}} \oplus \mathrm{F} D_{\theta_{2}} \oplus \mu
$$

where $\mu$ is a $\phi$-invariant normal subbundle of $\mathcal{N}$.

\section{Cohomology Class for Bislant Submanifolds of Nearly Trans- Sasakian Manifolds}

Chen [21] introduces a canonical de Rham cohomology class for a closed CR-submanifold in a Kähler manifold. So, in this section, we discuss the de Rham cohomology class for a closed bislant submanifold of a nearly trans-Sasakian manifold $(\overline{\mathcal{N}}, \phi, \xi, \eta, g)$ with minimal horizontal distribution $\left(D_{\theta_{1}} \oplus\{\xi\}\right)$. We put $\operatorname{dim}(\mathcal{N})=m$ and $\operatorname{dim}\left(D_{\theta_{1}} \oplus\{\xi\}\right)=2 a+1$. Let us assume the following:

(i) $\left\{\mathscr{E}_{1}, \cdots, \mathscr{E}_{a}, \mathscr{E}_{a+1}=\sec \theta_{1} \mathrm{P} \mathscr{E}_{1}, \cdots, \mathscr{E}_{2 a}=\sec \theta_{1} \mathrm{P} \mathscr{E}_{a}\right.$, $\mathscr{E}_{2 a+1}=\xi, \mathscr{E}_{2 a+2}=\mathscr{E}_{1}^{*}, \cdots, \mathscr{E}_{2 a+b+1}=\mathscr{E}_{b}^{*}, \mathscr{E}_{2 a+b+2}=$ $\mathscr{E}_{b+1}^{*}=\sec \theta_{2} \mathrm{P} \mathscr{E}_{1}^{*}, \cdots, \mathscr{E}_{m}=\mathscr{E}_{2 a+2 b+1}=\mathscr{E}_{2 b}^{*}=\sec \theta_{2} \mathrm{P}$ $\left.\mathscr{E}_{b}^{*}\right\}$ is a local orthonormal frame of $\mathcal{N}$

(ii) $\left\{\mathscr{E}_{1}, \cdots, \mathscr{E}_{a}, \mathscr{E}_{a+1}=\sec \theta_{1} \mathrm{P} \mathscr{E}_{1}, \cdots, \mathscr{E}_{2 a}=\sec \theta_{1} \mathrm{P} \mathscr{E}_{a}\right.$ $\left.\mathscr{E}_{2 a+1}=\xi\right\}$ is a local orthonormal frame of $\left(D_{\theta_{1}} \oplus\{\right.$ $\xi\})$

(iii) $\left\{\mathscr{E}_{2 a+2}=\mathscr{E}_{1}^{*}, \cdots, \mathscr{E}_{2 a+b+1}=\mathscr{E}_{b}^{*}, \mathscr{E}_{2 a+b+2}=\mathscr{E}_{b+1}^{*}=\mathrm{sec}\right.$ $\left.\theta_{2} \mathrm{P} \mathscr{E}_{1}^{*}, \cdots, \mathscr{E}_{m}=\mathscr{E}_{2 a+2 b+1}=\mathscr{E}_{2 b}^{*}=\sec \theta_{2} \mathrm{P} \mathscr{E}_{b}^{*}\right\}$ is a local orthonormal frame of $D_{\theta_{2}}$

We choose $\varsigma^{1}, \cdots, \varsigma^{2 a+1}, \varsigma^{2 a+2}, \cdots, \varsigma^{m}$ as the dual frame of 1 -forms to the above local orthonormal frame. Then, we define a $(2 a+1)$-form $\omega$ on $\mathcal{N}$ by $\omega=\varsigma^{1} \wedge \varsigma^{2} \wedge \cdots \wedge \varsigma^{2 a+1}$. It is globally defined on $\mathscr{N}$. In the same way, we again define 
a $(m-2 a-1)$-form $\Omega$ on $\mathcal{N}$ by $\Omega=\varsigma^{2 a+2} \wedge \varsigma^{2 a+3} \wedge \cdots \wedge \varsigma^{m}$, which is globally defined on $\mathcal{N}$.

We prepare some preliminary lemmas.

Lemma 9. Let $\mathcal{N}$ be a submanifold of an arbitrary nearly trans-Sasakian manifold $\overline{\mathcal{N}}$, then

$$
\begin{array}{r}
\nabla_{X} P Y-A_{F Y} X-P \nabla_{Y} X-2 B h(X, Y)+\nabla_{Y} P X-A_{F X} Y-P \nabla_{X} Y \\
=\alpha(2 g(X, Y) \xi-\eta(Y) X-\eta(X) Y)-\beta(\eta(Y) P X+\eta(X) P Y),
\end{array}
$$

$$
\begin{aligned}
& h(X, P Y)+\nabla_{X}^{\perp} F Y-F \nabla_{X} Y-2 \mathscr{C} h(X, Y)+h(Y, P X)+\nabla Y^{\perp} F X-F \nabla_{Y} X \\
& \quad=-\beta(\eta(Y) F X+\eta(X) F Y),
\end{aligned}
$$

for any $X, Y \in T \mathcal{N}$.

Proof. For any vector fields $X, Y \in T \mathcal{N}$, making use of the structure equation and (2), we obtain

$$
\begin{aligned}
& \bar{\nabla}_{X} \phi Y-\phi \bar{\nabla}_{X} Y+\bar{\nabla}_{Y} \phi X-\phi \bar{\nabla}_{Y} X=\alpha(2 g(X, Y) \xi-\eta(Y) X-\eta(X) Y) \\
& \quad-\beta(\eta(Y) \phi X+\eta(X) \phi Y),
\end{aligned}
$$

which gives

$$
\begin{aligned}
\nabla_{X} \mathrm{P} Y & +h(\mathrm{P} Y, X)-A_{\mathrm{F} Y} X+\nabla_{X}^{\perp} \mathrm{F} Y-\mathrm{P} \nabla_{X} Y-2 \mathrm{~B} h(X, Y) \\
& -\mathrm{F} \nabla_{X} Y-2 \mathscr{C} h(X, Y)+\nabla_{X} \mathrm{P} Y+h(\mathrm{P} X, Y)-A_{\mathrm{F} X} Y \\
& +\nabla_{Y}^{\perp} \mathrm{F} X-\mathrm{P} \nabla_{Y} X-\mathrm{F} \nabla_{Y} X \\
= & \alpha(2 g(X, Y) \xi-\eta(Y) X-\eta(X) Y) \\
& -\beta(\eta(Y) \mathrm{P} X+\eta(X) \mathrm{P} Y+\eta(Y) \mathrm{F} X+\eta(X) \mathrm{F} Y) .
\end{aligned}
$$

Comparing the tangential and normal components of the above equation, we get the desired relations (15) and (16).

The next lemma gives the integrability condition of slant distribution $D_{\theta_{2}}$.

Lemma 10. Let $\mathcal{N}$ be a bislant submanifold of an arbitrary nearly trans-Sasakian manifold $(\overline{\mathcal{N}}, \phi, \xi, \eta, g)$. Then, slant distribution $D_{\theta_{2}}$ is integrable if and only if

$$
-2 F \nabla_{Y} X+h(X, P Y)+h(Y, P X)-2 \mathscr{C} h(X, Y)+\nabla_{X}^{\perp} F Y+\nabla_{Y}^{\perp} F X \in F D_{\theta_{2}},
$$

for any $X, Y \in D_{\theta_{2}}$.

Proof. Making use of Lemma 9, we obtain

$$
\begin{aligned}
g(\mathrm{~F}[X, Y], \mathrm{FZ})= & -2\left\{g\left(\mathrm{~F}_{Y} X, \mathrm{~F} Z\right)+g(h(X, \mathrm{P} Y), \mathrm{F} Z)\right. \\
& +g(h(Y, \mathrm{P} X), \mathrm{F} Z)-g(2 \mathscr{C} h(X, Y), \mathrm{FZ}) \\
& \left.+g\left(\nabla_{X}^{\perp} \mathrm{F} Y, \mathrm{~F} Z\right)+g\left(\nabla_{Y}^{\perp} \mathrm{F} X, \mathrm{~F} Z\right)\right\},
\end{aligned}
$$

for any $Z \in\left(D_{\theta_{1}} \oplus\{\xi\}\right)$. Thus, the assertion follows from the fact that $\mathrm{F} D_{\theta_{1}}$ and $\mathrm{F} D_{\theta_{2}}$ are mutually perpendicular. In this way, we proved the integrability condition of slant distribution $D_{\theta_{2}}$.

We prove the following.

Theorem 11. For any closed bislant submanifold $\mathcal{N}$ of an arbitrary nearly trans-Sasakian manifold $(\overline{\mathcal{N}}, \phi, \xi, \eta, g)$ with minimal $\left(D_{\theta_{1}} \oplus\{\xi\}\right)$ and

$$
-2 F \nabla_{Y} X+h(X, P Y)+h(Y, P X)-2 \mathscr{C} h(X, Y)+\nabla_{X}^{\perp} F Y+\nabla_{Y}^{\perp} F X \in F D_{\theta_{2}},
$$

for any $X, Y \in D_{\theta_{2}}$, the $(2 a+1)$-form $\omega$ is closed and defines a canonical de Rham cohomology class $[\omega] \in H^{2 a+1}($ $\mathcal{N}, \mathbb{R})$, where $\operatorname{dim}\left(D_{\theta_{1}} \oplus\{\xi\}\right)=2 a+1$.

Moreover, the cohomology group $H^{2 a+1}(\mathcal{N}, \mathbb{R})$ is nontrivial if $D_{\theta_{2}}$ is minimal and $\left(D_{\theta_{1}} \oplus\{\xi\}\right)$ is integrable.

Proof. From the definition of $\omega$, we have $d \omega=\sum_{i=1}^{2 a+1}(-1)^{i-1}$ $\varsigma^{1} \wedge \cdots \wedge d \varsigma^{i} \wedge \cdots \wedge \varsigma^{2 a+1}$, which implies that $d \omega=0$ if and only if

$$
\begin{aligned}
& d \omega\left(X_{2}, Y_{2}, X_{1}, \cdots, X_{2 a}\right)=0, \\
& d \omega\left(X_{2}, X_{1}, \cdots, X_{2 a+1}\right)=0
\end{aligned}
$$

for any $X_{2}, Y_{2} \in D_{\theta_{2}}$ and $X_{1}, \cdots, X_{2 a+1} \in\left(D_{\theta_{1}} \oplus\{\xi\}\right)$. Thus, by simple computation, we find that (22) is satisfied if and only if $D_{\theta_{2}}$ is integrable. On the other hand, (23) is satisfied if and only if $\left(D_{\theta_{1}} \oplus\{\xi\}\right)$ is minimal. However, the integrability condition of $D_{\theta_{2}}$ holds due to Lemma 10 , and by the hypothesis of the theorem, we have $\left(D_{\theta_{1}} \oplus\{\xi\}\right)$ is minimal. Hence, the form $\omega$ is closed. It defines a canonical de Rham cohomology class $[\omega] \in H^{2 a+1}(\mathcal{N}, \mathbb{R})$.

Next, we prove that the cohomology class $[\omega]$ is nontrivial. Since $D_{\theta_{2}}$ is minimal and $\left(D_{\theta_{1}} \oplus\{\xi\}\right)$ is integrable, then in this case, we need to show that $\omega$ is harmonic. By definition of $\Omega$ and the similar argument for $\omega$, we see that $d \Omega=0$, that is, $\Omega$ is closed, if $\left(D_{\theta_{1}} \oplus\{\xi\}\right)$ is integrable and $D_{\theta_{2}}$ is minimal. This further proves that $\delta \omega=0$, that is, $\omega$ is coclosed. From $d \omega=0, \delta \omega=0$, and $\mathscr{N}$ is a closed submanifold, we deduce that $\omega$ is harmonic $(2 a+1)$-form. Hence, the cohomology group $H^{2 a+1}(\mathcal{N}, \mathbb{R})$ is nontrivial if $D_{\theta_{2}}$ is minimal and $\left(D_{\theta_{1}} \oplus\{\xi\}\right)$ is integrable.

\section{Warped Product Bislant Submanifolds}

Definition 12 (see [22]). Let $\left(\mathcal{N}_{1}, g_{1}\right)$ and $\left(\mathcal{N}_{2}, g_{2}\right)$ be two Riemannian manifolds and $f>0$ be a differentiable function on $\mathcal{N}_{1}$. Consider two projections on $\mathcal{N}_{1} \times \mathcal{N}_{2}, \rho: \mathcal{N}_{1} \times$ $\mathcal{N}_{2} \longrightarrow \mathcal{N}_{1}$ and $\delta: \mathcal{N}_{1} \times \mathcal{N}_{2} \longrightarrow \mathcal{N}_{2}$. The projection maps given by $\rho(p, q)=p$ and $\delta(p, q)=q$ for $(p, q) \in \mathcal{N}_{1} \times \mathcal{N}_{2}$. Then, the warped product $\mathcal{N}=\mathcal{N}_{1} \times{ }_{f} \mathcal{N}_{2}$ is the product 
manifold $\mathcal{N}_{1} \times \mathscr{N}_{2}$ equipped with the Riemannian structure such that

$$
g(X, Y)=g_{1}\left(\rho_{*} X, \rho_{*} Y\right)+(f \circ \rho)^{2} g_{2}\left(\delta_{*} X, \delta_{*} Y\right),
$$

for any $X, Y \in T \mathcal{N}$, where $*$ is the symbol for the tangent maps. The function $f$ is called the warping function of $\mathcal{N}$.

Example 13. A surface of revolution is a warped product manifold.

Example 14. The standard space-time models of the universe are warped products as the simplest models of neighbourhoods of stars and black holes.

Remark 15. In particular, a warped product manifold is said to be trivial if its warping function is constant. In such a case, we call the warped product manifold a Riemannian product manifold. If $\mathcal{N}=\mathscr{N}_{1} \times{ }_{f} \mathcal{N}_{2}$ is a warped product manifold, then $\mathscr{N}_{1}$ is totally geodesic and $\mathscr{N}_{2}$ is totally umbilical submanifold of $\mathcal{N}$ [22].

Let $\mathcal{N}=\mathscr{N}_{1} \times{ }_{f} \mathcal{N}_{2}$ be a warped product manifold with a warping function $f$. Then,

$$
\nabla_{X} Z=\nabla_{Z} X=(X \ln f) Z
$$

for any $X \in T \mathcal{N}_{1}$ and $Z \in T \mathcal{N}_{2}$, where $\nabla \ln f$ is the gradient of $\ln f$ and $\nabla$ and $\nabla^{\mathcal{N}_{2}}$ denote the Levi-Civita connections on $\mathcal{N}$ and $\mathcal{N}_{2}$, respectively.

The definition of warped product bislant submanifolds in a nearly trans-Sasakian manifold is as follows.

Definition 16. A warped product $\mathcal{N}_{1} \times{ }_{f} \mathcal{N}_{2}$ of two slant submanifolds $\mathcal{N}_{1}$ and $\mathcal{N}_{2}$ of a nearly trans-Sasakian manifold $\overline{\mathcal{N}}$ is called a warped product bislant submanifold.

Remark 17. A warped product bislant submanifold $\mathscr{N}_{1} \times_{f}$ $\mathcal{N}_{2}$ is called proper if $\mathcal{N}_{1}$ and $\mathcal{N}_{2}$ are proper slant in $\overline{\mathcal{N}}$. Otherwise, the warped product bislant submanifold $\mathcal{N}_{1} \times_{f}$ $\mathcal{N}_{2}$ is called nonproper.

For a warped product bislant submanifold in a nearly trans-Sasakian manifold such that $\xi \in T \mathcal{N}_{1}$, we have the following result.

Theorem 18. Let $\mathcal{N}=\mathscr{N}_{1} \times{ }_{f} \mathcal{N}_{2}$ be a warped product bislant submanifold with bislant angles $\left\{\theta_{1}, \theta_{2}\right\}$ in a nearly transSasakian manifold $\overline{\mathcal{N}}$ such that $\xi \in T \mathcal{N}_{1}$. If, for any $X_{1} \in T$ $\mathcal{N}_{1}$ and $X_{2}, Y_{2} \in T \mathcal{N}_{2}$,

$$
g\left(h\left(X_{1}, X_{2}\right), F Y_{2}\right)=g\left(h\left(X_{1}, Y_{2}\right), F X_{2}\right),
$$

holds, then one of the following cases must occur: (i) $\mathcal{N}$ is a warped product pseudoslant submanifold such that $\mathcal{N}_{2}$ is a totally real submanifold $\mathcal{N}^{\perp}$ of $\overline{\mathcal{N}}$

(ii) If $\overline{\mathcal{N}}$ is nearly Sasakian manifold, that is, $\beta=0$, then $\mathcal{N}$ is a Riemannian product

(iii) If $\beta \neq 0$, then $\beta \eta\left(X_{1}\right)=-\left(X_{1} \ln f\right)$

Proof. For any vector fields $X_{1} \in T \mathcal{N}_{1}$ and $X_{2}, Y_{2} \in T \mathcal{N}_{2}$, we have

$$
\begin{aligned}
g\left(h\left(X_{1}, X_{2}\right), \mathrm{F} Y_{2}\right)= & g\left(\bar{\nabla}_{X_{1}} X_{2}, \phi Y_{2}\right)-g\left(\nabla_{X_{1}} X_{2}, \mathrm{P} Y_{2}\right) \\
= & g\left(\left(\bar{\nabla}_{X_{1}} \phi\right) X_{2}, Y_{2}\right)-g\left(\bar{\nabla}_{X_{1}} \phi X_{2}, Y_{2}\right) \\
& -\left(X_{1} \ln f\right) g\left(X_{2}, \mathrm{P} Y_{2}\right) .
\end{aligned}
$$

On the other hand, we have

$$
\begin{aligned}
g\left(h\left(X_{1}, X_{2}\right), \mathrm{F} Y_{2}\right)= & g\left(\bar{\nabla}_{X_{2}} X_{1}, \phi Y_{2}\right)-g\left(\nabla_{X_{2}} X_{1}, \mathrm{P} Y_{2}\right) \\
= & g\left(\left(\bar{\nabla}_{X_{2}} \phi\right) X_{1}, Y_{2}\right)-g\left(\bar{\nabla}_{X_{2}} \phi X_{1}, Y_{2}\right) \\
& -\left(X_{1} \ln f\right) g\left(X_{2}, \mathrm{P} Y_{2}\right) .
\end{aligned}
$$

By adding (27) and (28), we get

$$
\begin{aligned}
2 g\left(h\left(X_{1}, X_{2}\right), \mathrm{FY} Y_{2}\right)= & g\left(h\left(X_{1}, Y_{2}\right), \mathrm{F} X_{2}\right)+g\left(h\left(X_{2}, Y_{2}\right), \mathrm{F} X_{1}\right) \\
& -(\operatorname{Pln} f) g\left(X_{2}, Y_{2}\right)-\left(X_{1} \ln f\right) g\left(X_{2}, \mathrm{PY}_{2}\right) \\
& -\alpha \eta\left(X_{1}\right) g\left(X_{2}, Y_{2}\right)+\beta \eta\left(X_{1}\right) g\left(\mathrm{P}_{2}, Y_{2}\right) .
\end{aligned}
$$

Interchanging $X_{2}$ by $Y_{2}$ in (29), we find

$$
\begin{aligned}
2 g\left(h\left(X_{1}, Y_{2}\right), \mathrm{FX}_{2}\right)= & g\left(h\left(X_{1}, X_{2}\right), \mathrm{F} Y_{2}\right)+g\left(h\left(X_{2}, Y_{2}\right), \mathrm{FX}_{1}\right) \\
& -(\operatorname{Pln} f) g\left(X_{2}, Y_{2}\right)-\left(X_{1} \ln f\right) g\left(Y_{2}, \mathrm{P} X_{2}\right) \\
& -\alpha \eta\left(X_{1}\right) g\left(X_{2}, Y_{2}\right)+\beta \eta\left(X_{1}\right) g\left(\mathrm{P}_{2}, X_{2}\right) .
\end{aligned}
$$

By subtracting (30) from (29) and by applying our assumption, we obtain

$$
g\left(\mathrm{P} X_{2}, Y_{2}\right)\left[\left(X_{1} \ln f\right)+\beta \eta\left(X_{1}\right)\right]=0 .
$$

For $Y_{2}=\mathrm{P} Y_{2}$, we get

$$
\cos ^{2} \theta_{2} g\left(Y_{2}, X_{2}\right)\left[\left(X_{1} \ln f\right)+\beta \eta\left(X_{1}\right)\right]=0 .
$$

From the last expression, any one of the following holds: if $\beta=0$, then $f$ is constant, or if $\beta \neq 0$, then $\beta \eta\left(X_{1}\right)=-\left(X_{1}\right.$ $\ln f$ ) or $\theta_{2}=\pi / 2$. Thus, our assertions follow.

Now, we have the following theorem for a warped product bislant submanifold in a nearly trans-Sasakian manifold such that $\xi \in T \mathcal{N}_{2}$. 
Theorem 19. Let $\mathcal{N}=\mathcal{N}_{1} \times{ }_{f} \mathcal{N}_{2}$ be a warped product bislant submanifold with bislant angles $\left\{\theta_{1}, \theta_{2}\right\}$ in a nearly transSasakian manifold $\overline{\mathcal{N}}$ such that $\xi \in T \mathcal{N}_{2}$. If, for any $X_{1} \in T$ $\mathscr{N}_{1}$ and $X_{2}, Y_{2} \in T \mathscr{N}_{2}$,

$$
g\left(h\left(X_{1}, X_{2}\right), F Y_{2}\right)=g\left(h\left(X_{1}, Y_{2}\right), F X_{2}\right)
$$

holds, then one of the following cases must occur:

(i) $\mathcal{N}$ is a warped product pseudoslant submanifold such that $\mathcal{N}_{2}$ is a totally real submanifold $\mathcal{N}^{\perp}$ of $\overline{\mathcal{N}}$

(ii) $\mathcal{N}$ is a Riemannian product

Proof. For any vector fields $X_{1} \in T \mathcal{N}_{1}$ and $X_{2}, Y_{2} \in T \mathcal{N}_{2}$, we have

$$
\begin{aligned}
g\left(h\left(X_{1}, X_{2}\right), \mathrm{FY}_{2}\right) & =g\left(\bar{\nabla}_{X_{1}} X_{2}, \phi Y_{2}\right)-g\left(\nabla_{X_{1}} X_{2}, \mathrm{P} Y_{2}\right) \\
& =g\left(\left(\bar{\nabla}_{X_{1}} \phi\right) X_{2}, Y_{2}\right)-g\left(\bar{\nabla}_{X_{1}} \phi X_{2}, Y_{2}\right) .
\end{aligned}
$$

On the other hand, we have

$$
\begin{aligned}
g\left(h\left(X_{1}, X_{2}\right), \mathrm{F} Y_{2}\right) & =g\left(\bar{\nabla}_{X_{2}} X_{1}, \phi Y_{2}\right)-g\left(\nabla_{X_{2}} X_{1}, \mathrm{P} Y_{2}\right) \\
& =g\left(\left(\bar{\nabla}_{X_{2}} \phi\right) X_{1}, Y_{2}\right)-g\left(\bar{\nabla}_{X_{2}} \phi X_{1}, Y_{2}\right) .
\end{aligned}
$$

By adding (34) and (35), we get

$$
\begin{aligned}
2 g\left(h\left(X_{1}, X_{2}\right), \mathrm{F} Y_{2}\right)= & g\left(h\left(X_{1}, Y_{2}\right), \mathrm{F} X_{2}\right)+g\left(h\left(X_{2}, Y_{2}\right), \mathrm{F} X_{1}\right) \\
& -(\mathrm{P} \ln f) g\left(X_{2}, Y_{2}\right)-\left(X_{1} \ln f\right) g\left(X_{2}, \mathrm{P} Y_{2}\right) .
\end{aligned}
$$

Interchanging $X_{2}$ by $Y_{2}$ in (36), we find

$$
\begin{aligned}
2 g\left(h\left(X_{1}, Y_{2}\right), \mathrm{F}_{2}\right)(37) & =g\left(h\left(X_{1}, X_{2}\right), \mathrm{F} Y_{2}\right)+g\left(h\left(X_{2}, Y_{2}\right), \mathrm{F} X_{1}\right) \\
- & (\operatorname{Pln} f) g\left(X_{2}, Y_{2}\right)-\left(X_{1} \ln f\right) g\left(Y_{2}, \mathrm{P} X_{2}\right)
\end{aligned}
$$

By subtracting (37) from (36) and by applying our assumption, we obtain

$$
\left(X_{1} \ln f\right) g\left(\mathrm{P} X_{2}, Y_{2}\right)=0
$$

For $Y_{2}=\mathrm{PY}_{2}$, we get

$$
\cos ^{2} \theta_{2}\left(X_{1} \ln f\right)\left[g\left(Y_{2}, X_{2}\right)-\eta\left(X_{2}\right) \eta\left(Y_{2}\right)\right]=0 \text {. }
$$

Therefore, either $f$ is constant or $\cos \theta_{2}=0$ holds. Consequently, either $\mathcal{N}$ is a Riemannian product or $\theta_{2}=\pi / 2$. In the latter case, $\mathcal{N}$ is a warped product pseudoslant submanifold.

We give some nontrivial examples of warped product bislant submanifold of the form $\mathcal{N}=\mathcal{N}_{\theta} \times{ }_{f} \mathcal{N}_{\perp}$ whose bislant angles $\theta_{1} \neq 0, \pi / 2$ and $\theta_{2}=\pi / 2$. Such warped product bislant submanifolds are called pseudoslant submanifolds.

Example 20. Let $\mathbb{C}^{4}$ be the complex Euclidean space with its usual Kähler structure and the real global coordinates $\left(x_{1}\right.$, $\left.y_{1}, x_{2}, y_{2}, x_{3}, y_{3}, x_{4}, y_{4}\right)$ and $\overline{\mathcal{N}}=\mathbb{R} \times{ }_{f} \mathbb{C}^{4}$ be a warped product manifold between the product real line of $\mathbb{R}$ and the complex space $\mathbb{C}^{4}$. Let $<$, > be the Euclidean metric tensor of $\mathbb{R}^{9}$. An almost contact structure $\phi$ of $\overline{\mathcal{N}}$ is defined by

$$
\phi\left(\frac{\partial}{\partial x_{i}}\right)=\frac{\partial}{\partial y_{i}}, \phi\left(\frac{\partial}{\partial y_{j}}\right)=-\frac{\partial}{\partial x_{j}}, \phi\left(\frac{\partial}{\partial t}\right)=0, \quad 1 \geq i, j \geq 4
$$

such that

$$
\xi=e^{t}\left(\frac{\partial}{\partial t}\right), \eta=e^{t} d t, g=e^{t}<,>
$$

On the other hand, we define a submanifold $\mathcal{N}$ by immersion $g$ as follows:

$g(u, v, w, s, t)=(u, v, 0,0, v \cos r, v \sin r, s \cos w, s \sin w, t)$

Therefore, it is easy to choose tangent bundle of $\mathcal{N}$ which is spanned by the following:

$$
\begin{aligned}
& X_{1}=\frac{\partial}{\partial x_{1}}, X_{2}=\cos r \frac{\partial}{\partial y_{1}}+\sin r \frac{\partial}{\partial y_{2}} \\
& X_{3}=\cos w \frac{\partial}{\partial y_{3}}+\sin w \frac{\partial}{\partial y_{4}}, X_{5}=\frac{\partial}{\partial z}
\end{aligned}
$$

Thus, $D_{\theta_{1}}=\operatorname{Span}\left\{X_{1}, X_{2}\right\}$ is a slant distribution with slant angle $\pi / 4$. Also, it is easy to verify that $D_{\theta_{2}}=\operatorname{Span}\left\{X_{3}\right.$ ,$\left.X_{4}\right\}$ is a totally real distribution. Hence, the submanifold $\mathcal{N}$ defined by $f$ is a bislant submanifold, which is tangent to the structure vector $\xi$ and whose bislant angles satisfy $\theta_{1}$ $\neq 0, \pi / 2$ and $\theta_{2}=\pi / 2$. It is easy to check that the distributions $D_{\theta_{1}}$ and $D_{\theta_{2}}$ are integrable. Then, it can be verified that $\mathcal{N}=\mathcal{N}_{\theta} \times{ }_{f} \mathcal{N}_{\perp}$ is a warped product bislant submanifold of $\overline{\mathcal{N}}$ with warping function $f=e^{t}, t \in \mathbb{R}$.

Example 21. We consider any submanifold $\mathcal{N}$ in a nearly trans-Sasakian manifold $\mathbb{R}^{7}$

$$
\mathfrak{f}(u, v, w, q)=(u \cos v, w \cos v, u \sin v, w \sin v, w-u, w+u, q) .
$$


The tangent bundle of $\mathscr{N}$ is spanned by

$$
\begin{gathered}
\mathscr{E}_{1}=\cos v \frac{\partial}{\partial x_{1}}+\sin v \frac{\partial}{\partial x_{2}}-\frac{\partial}{\partial x_{3}}+\frac{\partial}{\partial y_{3}}, \\
\mathscr{E}_{2}=-u \sin v \frac{\partial}{\partial x_{1}}+u \cos v \frac{\partial}{\partial x_{2}}-w \sin v \frac{\partial}{\partial y_{1}}+w \cos v \frac{\partial}{\partial y_{2}}, \\
\mathscr{E}_{3}=\frac{\partial}{\partial x_{3}}+\cos v \frac{\partial}{\partial y_{1}}+\sin v \frac{\partial}{\partial y_{2}}+\frac{\partial}{\partial y_{3}}, \\
\mathscr{E}_{4}=\frac{\partial}{\partial q}
\end{gathered}
$$

Furthermore, we have

$$
\begin{gathered}
\phi \mathscr{E}_{1}=\cos v \frac{\partial}{\partial y_{1}}+\sin v \frac{\partial}{\partial y_{2}}-\frac{\partial}{\partial y_{3}}-\frac{\partial}{\partial x_{3}}, \\
\phi \mathscr{C}_{2}=-u \sin v \frac{\partial}{\partial y_{1}}+u \cos v \frac{\partial}{\partial y_{2}}+w \sin v \frac{\partial}{\partial x_{1}}-w \cos v \frac{\partial}{\partial x_{2}}, \\
\phi \mathscr{C}_{3}=\frac{\partial}{\partial y_{3}}-\cos v \frac{\partial}{\partial x_{1}}-\sin v \frac{\partial}{\partial x_{2}}-\frac{\partial}{\partial x_{3}}, \\
\phi \mathscr{C}_{4}=0 .
\end{gathered}
$$

It is easy to check that $\phi \mathscr{E}_{2}$ is orthogonal to $T \mathcal{N}$. Then, the proper slant and anti-invariant distributions of $\mathcal{N}$ are respectively defined by $D_{\theta}=\operatorname{Span}\left\{\mathscr{E}_{1}, \mathscr{E}_{3}\right\}$ with slant angle $\theta=\arccos (1 / 3)$ and $D_{\perp}=\operatorname{Span}\left\{\mathscr{E}_{2}\right\}$. Also, $\mathscr{E}_{4}=\xi$ is tangent to $D_{\theta}$. Hence, $\mathfrak{f}$ defines a proper 4 -dimensional pseudoslant submanifold (bislant submanifold with bislant angles \{ $\arccos (1 / 3), \pi / 2\}) \mathcal{N}$ in $\mathbb{R}^{7}$. It is easy to check that the distributions $D_{\theta} \oplus\{\xi\}$ and $D_{\perp}$ are integrable.

Now, we assume that $\mathcal{N}_{\theta}$ and $\mathcal{N}_{\perp}$ are the integral manifolds of $D_{\theta}$ and $D_{\perp}$, respectively. Then, it follows from Definition 12 and (44) that the induced metric tensor $g$ of $\mathcal{N}$ is given by

$$
\begin{aligned}
g= & \left(\cos ^{2} v+\sin ^{2} v+2\right) d u^{2}+\left(u^{2} \sin ^{2} v+u^{2} \cos ^{2} v+w^{2} \sin ^{2} v+w^{2} \cos ^{2} v\right) d v^{2} \\
& +\left(\cos ^{2} v+\sin ^{2} v+2\right) d w^{2}+d q^{2}=3\left(d u^{2}+d w^{2}\right)+d q^{2}+\left(u^{2}+w^{2}\right) d v^{2} \\
= & g_{1}+g_{2},
\end{aligned}
$$

where $g_{1}=3\left(d u^{2}+d w^{2}\right)+d q^{2}$ and $g_{2}=\left(u^{2}+w^{2}\right) d v^{2}$ are respectively the metric tensors of $\mathcal{N}_{\theta}$ and $\mathcal{N}_{\perp}$. As a consequence, $\mathcal{N}=\mathscr{N}_{\theta} \times{ }_{f} \mathcal{N}_{\perp}$ is a warped product pseudoslant submanifold of $\mathbb{R}^{7}$ with a warping function, that is, $f=$ $\sqrt{u^{2}+w^{2}}$ such that $\xi$ is tangent to $\mathcal{N}_{\theta}$.

\section{Doubly Warped Product Bislant Submanifolds}

In general, doubly warped products can be considered as a generalization of warped products.
Definition 22 (see $[23,24])$. Let $\left(\mathcal{N}_{1}, g_{1}\right)$ and $\left(\mathcal{N}_{2}, g_{2}\right)$ be Riemannian manifolds. A doubly warped product $(\mathcal{N}, g)$ is a product manifold which is of the form $\mathscr{N}={ }_{f_{2}} \mathcal{N}_{1} \times{ }_{f_{1}} \mathcal{N}$ with the metric $g=f_{1}^{2} g_{1} \oplus f_{2}^{2} g_{2}$, where $f_{1}: \mathcal{N}_{1} \times \mathcal{N}_{2} \longrightarrow(0$ $, \infty)$ and $f_{2}: \mathscr{N}_{1} \times \mathscr{N}_{2} \longrightarrow(0, \infty)$ are smooth maps. More precisely, if $\rho: \mathcal{N}_{1} \times \mathscr{N}_{2} \longrightarrow \mathcal{N}_{1}$ and $\delta: \mathcal{N}_{1} \times \mathcal{N}_{2} \longrightarrow \mathcal{N}_{2}$ are natural projections, the metric $g$ is defined by

$$
g(X, Y)=\left(f_{2} \circ \delta\right)^{2} g_{1}\left(\rho_{*} X, \rho_{*} Y\right)+\left(f_{1} \circ \rho\right)^{2} g_{2}\left(\delta_{*} X, \delta_{*} Y\right),
$$

for any $X, Y \in T \mathcal{N}$, where $*$ is the symbol for the tangent maps. The functions $f_{1}$ and $f_{2}$ are called the warping functions of $\mathcal{N}$.

Remark 23. If we assume

(i) either $f_{1} \equiv 1$ or $f_{2} \equiv 1$, but not both, then we obtain a warped product

(ii) both $f_{1} \equiv 1$ and $f_{2} \equiv 1$, then we have a product manifold

(iii) neither $f_{1}$ nor $f_{2}$ is constant, then we have a nontrivial doubly warped product

For doubly warped product manifold $\mathscr{N}={ }_{f_{2}} \mathcal{N}_{1} \times{ }_{f_{1}} \mathcal{N}$ with warping functions $f$ and $g$, we have the following:

$$
\nabla_{Y} X=\nabla_{X} Y=\left(Y \ln f_{1}\right) X+\left(X \ln f_{2}\right) Y,
$$

for any $X \in T \mathcal{N}_{1}$ and $Y \in T \mathcal{N}_{2}$.

Now, we define the notion of doubly warped product bislant submanifolds in nearly trans-Sasakian manifolds as follows.

Definition 24. The doubly warped product of two slant submanifolds, $f_{2} \mathcal{N}_{1} \times_{f_{1}} \mathcal{N}_{2}$, is called the doubly warped product bislant submanifold of slant submanifolds $\mathcal{N}_{1}$ and $\mathcal{N}_{2}$ with slant angles $\theta_{1}$ and $\theta_{2}$, respectively, of a nearly transSasakian manifold with warping functions $f_{1}$ and $f_{2}$ if only depend on the points of $\mathcal{N}_{1}$ and $\mathcal{N}_{2}$, respectively.

First we have the following theorem for doubly warped product submanifolds $\mathscr{N}={ }_{f_{2}} \mathcal{N}_{1} \times_{f_{1}} \mathcal{N}_{2}$ in nearly transSasakian manifolds such that $\xi \in T \mathcal{N}_{1}$.

Theorem 25. Let $\mathcal{N}={ }_{f_{2}} \mathcal{N}_{1} \times{ }_{f_{1}} \mathcal{N}_{2}$ be a doubly warped product submanifold in a nearly trans-Sasakian manifold $\overline{\mathcal{N}}$, where $\mathcal{N}_{1}$ and $\mathcal{N}_{2}$ are Riemannian submanifolds of $\overline{\mathcal{N}}$ and $\xi \in T \mathcal{N}_{1}$. Then, $\mathcal{N}$ is a warped product bislant submanifold of type $\mathcal{N}_{1} \times{ }_{f_{1}} \mathcal{N}_{2}$ if and only if

$$
g(h(X, Y), F X)=g(h(X, X), F Y),
$$

for any $X \in T \mathcal{N}_{1}$ and $Y \in T \mathcal{N}_{2}$. 
Proof. From Lemma 9, we get

$$
\begin{aligned}
& \nabla_{X} \mathrm{P} Y-A_{\mathrm{FY}} X-\mathrm{P} \nabla_{Y} X-2 \mathbf{B} h(X, Y)+\nabla_{Y} \mathrm{P} X-A_{\mathrm{FX}} Y-\mathrm{P} \nabla_{X} Y \\
& \quad=-\alpha \eta(X) Y-\beta \eta(X) \mathrm{P} Y
\end{aligned}
$$

for any $X \in T \mathcal{N}_{1}$ and $Y \in T \mathcal{N}_{2}$. Applying (49), we derive

$$
\begin{gathered}
\left(\mathrm{P} Y \ln f_{2}\right) X-\left(Y \ln f_{2}\right) \mathrm{P} X-\left(X \ln f_{1}\right) \mathrm{P} Y+\left(\mathrm{P} X \ln f_{1}\right) Y \\
-A_{\mathrm{F} Y} X-2 \mathrm{~B} h(X, Y)-A_{\mathrm{F} X} Y=-\alpha \eta(X) Y-\beta \eta(X) \mathrm{P} Y .
\end{gathered}
$$

Taking the inner product with $X \in T \mathcal{N}_{1}$, we obtain

$\left(\mathrm{P} Y \ln f_{2}\right)\|X\|^{2}-g(h(X, X), \mathrm{F} Y)-2 g(\mathbf{B} h(X, Y), X)-g(h(Y, X), \mathrm{F} X)=0$.

Using relation (10) in the above equation, we get

$$
\left(\mathrm{P} Y \ln f_{2}\right)\|X\|^{2}=g(h(X, X), \mathrm{F} Y)-g(h(Y, X), \mathrm{F} X)=0 .
$$

Thus, from (54), we conclude that $\left(\mathrm{P} Y \ln f_{2}\right)=0$ if and only if

$$
g(h(X, Y), \mathrm{F} X)=g(h(X, X), \mathrm{F} Y)
$$

for any $X \in T \mathscr{N}_{1}$ and $Y \in T \mathcal{N}_{2} .\left(\mathrm{P} Y \ln f_{2}\right)=0$ shows that $f_{2}$ is constant, that is, $f_{2}$ depends only on the points of $\mathcal{N}_{1}$. Thus, it follows that $\mathcal{N}$ is a warped product bislant submanifold of type $\mathcal{N}_{1} \times{ }_{f_{1}} \mathcal{N}_{2}$. This proves the theorem completely.

Secondly, we prove the following theorem for doubly warped product bislant submanifolds $\mathcal{N}_{f_{2}} \mathcal{N}_{1} \times{ }_{f_{1}} \mathcal{N}_{2}$ in nearly trans-Sasakian manifolds such that $\xi \in T \mathcal{N}_{2}$.

Theorem 26. Let $\mathcal{N}={ }_{f_{2}} \mathcal{N}_{1} \times{ }_{f_{1}} \mathcal{N}_{2}$ be a doubly warped product bislant submanifold in a nearly trans-Sasakian manifold $\overline{\mathcal{N}}$, where $\mathcal{N}_{1}$ and $\mathcal{N}_{2}$ are proper slant submanifolds with respect to $\theta_{1}$ and $\theta_{2}$, respectively, and $\xi \in T \mathcal{N}_{2}$. Then, $\mathcal{N}$ is a warped product bislant submanifold of type $\mathcal{N}_{1} \times{ }_{f_{1}} \mathcal{N}_{2}$ if and only if

$$
g(h(X, Y), F Y)=g(h(Y, Y), F X)
$$

for any $X \in T \mathcal{N}_{2}$ and $Y \in T \mathcal{N}_{1}$.
Proof. For any vector fields $X \in T \mathcal{N}_{2}$ and $Y \in T \mathcal{N}_{1}$, we have

$$
\begin{aligned}
g(h(\mathrm{P} X, Y), \mathrm{F} Y) & =g\left(\bar{\nabla}_{Y} \mathrm{P} X, \phi Y\right)=-g\left(\phi \bar{\nabla}_{Y} \mathrm{P} X, Y\right) \\
& =g\left(\left(\bar{\nabla}_{Y} \phi\right) \mathrm{P} X, Y\right)-g\left(\bar{\nabla}_{Y} \phi \mathrm{P} X, Y\right) \\
& =-g\left(\left(\bar{\nabla}_{Y} \phi\right) Y, \mathrm{P} X\right)-g\left(\bar{\nabla}_{Y} \mathrm{P}^{2} X, Y\right)-g\left(\bar{\nabla}_{Y} \mathscr{F} \mathscr{P} X, Y\right) \\
& =\cos ^{2} \theta_{1} g\left(\nabla_{Y} X, Y\right)+g(h(Y, Y), \mathscr{F} \mathscr{P} X) \\
& =\cos ^{2} \theta_{1}\left(X \ln f_{2}\right)\|Y\|^{2}+g(h(Y, Y), \mathscr{F} \mathscr{P} X) .
\end{aligned}
$$

Replacing $X$ by $\mathrm{P} X$ in the last relation, we obtain

$$
\left(\mathrm{P} X \ln f_{2}\right)\|Y\|^{2}=g(h(Y, Y), \mathrm{F} X)-g(h(X, Y), \mathrm{F} Y) .
$$

Thus, from (54), we conclude that $\left(\mathrm{PX} \ln f_{2}\right)=0$ if and only if

$$
g(h(Y, Y), \mathrm{F} X)=g(h(X, Y), \mathrm{F} Y),
$$

for any $X \in T \mathcal{N}_{2}$ and $Y \in T \mathcal{N}_{1}$.

$\left(\mathrm{P} X \ln f_{2}\right)=0$ implies that $f_{2}$ is constant, that is, $f_{2}$ depends only on the points of $\mathscr{N}_{1}$. Hence, $\mathscr{N}$ is a warped product bislant submanifold of type $\mathcal{N}_{1} \times{ }_{f_{1}} \mathcal{N}_{2}$. This proves the theorem completely.

\section{Conclusion}

From Theorems 25 and 26, we conclude that there exist no doubly warped product bislant submanifolds in nearly trans-Sasakian manifolds, other than warped product bislant submanifolds, under some additional conditions.

\section{Some Applications of Theorem 25 for Different Kinds of Ambient Manifolds}

Let $\mathscr{N}={ }_{f_{2}} \mathcal{N}_{1} \times{ }_{f_{1}} \mathcal{N}_{2}$ be a doubly warped product submanifold, where $\mathcal{N}_{1}$ and $\mathcal{N}_{2}$ are Riemannian submanifolds of $\overline{\mathcal{N}}$ and $\xi \in T \mathcal{N}_{1}$. The following corollaries are the immediate consequences of Theorem 25.

Corollary 27. There does not exist any doubly warped product submanifold $\mathcal{N}={ }_{f_{2}} \mathcal{N}_{1} \times{ }_{f_{1}} \mathcal{N}_{2}$ in a nearly Sasakian manifold $\overline{\mathcal{N}}$, other than the warped product bislant submanifold, if and only if (50) holds.

Corollary 28. There does not exist a doubly warped product submanifold $\mathscr{N}={ }_{f_{2}} \mathcal{N}_{1} \times{ }_{f_{1}} \mathcal{N}_{2}$ in a nearly Kenmotsu manifold $\overline{\mathcal{N}}$, other than the warped product bislant submanifold, if and only if (50) holds.

Corollary 29. There does not exist a doubly warped product submanifold $\mathcal{N}={ }_{f_{2}} \mathcal{N}_{1} \times{ }_{f_{1}} \mathcal{N}_{2}$ in a nearly cosymplectic manifold $\overline{\mathcal{N}}$, other than the warped product bislant submanifold, if and only if (50) holds. 


\section{Some Applications of Theorem 26 for Different Kinds of Ambient Manifolds}

Let $\mathscr{N}={ }_{f_{2}} \mathcal{N}_{1} \times{ }_{f_{1}} \mathcal{N}_{2}$ be a doubly warped product bislant submanifold, where $\mathcal{N}_{1}$ and $\mathcal{N}_{2}$ are proper slant submanifolds with respect to $\theta_{1}$ and $\theta_{2}$, respectively, and $\xi \in T \mathcal{N}_{2}$. The following corollaries are the immediate consequences of Theorem 26.

Corollary 30. There is no doubly warped product bislant submanifold $\mathcal{N}={ }_{f_{2}} \mathcal{N}_{1} \times{ }_{f_{1}} \mathcal{N}_{2}$ in a nearly Sasakian manifold $\overline{\mathcal{N}}$, other than the warped product bislant submanifold, if and only if (56) holds.

Corollary 31. There is no doubly warped product bislant submanifold $\mathcal{N}={ }_{f_{2}} \mathcal{N}_{1} \times{ }_{f_{1}} \mathcal{N}_{2}$ in a nearly Kenmotsu manifold $\overline{\mathcal{N}}$ , other than the warped product bislant submanifold, if and only if (56) holds.

Corollary 32. There is no doubly warped product bislant submanifold $\mathcal{N}={ }_{f_{2}} \mathcal{N}_{1} \times{ }_{f_{1}} \mathcal{N}_{2}$ in a nearly cosymplectic manifold $\overline{\mathcal{N}}$, other than the warped product bislant submanifold, if and only if (56) holds.

\section{Data Availability}

There is no data used for this manuscript.

\section{Conflicts of Interest}

The authors declare no competing of interest.

\section{Authors' Contributions}

All authors have equal contribution and finalized.

\section{Acknowledgments}

The authors extend their appreciation to the Deanship of Scientific Research at King Khalid University for funding this work through a research group program under grant number R.G.P.2/74/42.

\section{References}

[1] A. N. Siddiqui, M. H. Shahid, and J. W. Lee, "Geometric inequalities for warped product bi-slant submanifolds with a warping function," Journal of Inequalities and Applications, vol. 2018, no. 1, 2018.

[2] B. Sahin, "Non-existence of warped product semi-slant submanifolds of Kaehler manifolds," Geometriae Dedicata, vol. 117, no. 1, pp. 195-202, 2006.

[3] S. Uddin, B.-Y. Chen, and F. R. Al-Solamy, "Warped product bi-slant immersions in Kaehler manifolds," Mediterranean Journal of Mathematics, vol. 14, no. 2, 2017.

[4] B.-Y. Chen, "Geometry of warped product CR-submanifolds in Kaehler manifolds," Monatshefte für Mathematik, vol. 133, no. 3, pp. 177-195, 2001.
[5] B.-Y. Chen, "Geometry of warped product CR-submanifolds in Kaehler manifolds, II," Monatshefte für Mathematik, vol. 134, no. 2, pp. 103-119, 2001.

[6] B. Sahin, "Notes on doubly warped and doubly twisted product CR-submanifolds of Kaehler manifolds," Matematiqki Vesnik, vol. 59, pp. 205-210, 2007.

[7] A. N. Siddiqui, "Warped product and doubly warped product bi-slant submanifolds in trans-Sasakian manifolds," JMI International Journal of Mathematical Sciences, vol. 9, pp. 15-27, 2018.

[8] A. Olteanu, "Contact CR-doubly warped product submanifolds in Kenmotsu space forms," Journal of Inequalities in Pure and Applied Mathematics, vol. 10, no. 4, 2009.

[9] F. R. Al-Solamy, M. F. Naghi, and S. Uddin, "Geometry of warped product pseudo-slant submanifolds of Kenmotsu manifolds," Quaestiones Mathematicae, vol. 42, no. 3, pp. 373-389, 2019.

[10] M. I. Munteanu, "Doubly warped product CR-submanifolds in locally conformal Kähler manifolds," Monatshefte für Mathematik, vol. 150, no. 4, pp. 333-342, 2007.

[11] S. Uddin, "On doubly warped and doubly twisted product submanifolds," International Electronic Journal of Geometry, vol. 3, no. 1, pp. 35-39, 2010.

[12] S. Uddin, I. Mihai, and A. Mihai, "On warped product bi-slant submanifolds of Kenmotsu manifolds," Arab Journal of Mathematical Sciences, vol. 27, no. 1, pp. 2-14, 2021.

[13] J. L. Cabrerizo, A. Carriazo, L. M. Fernandez, and M. Fernandez, "Semi-slant submanifolds of a Sasakian manifold," Geometriae Dedicata, vol. 78, no. 2, pp. 183-199, 1999.

[14] J. F. Nash, "The imbedding problem for Riemannian manifolds," Annals of Mathematics, vol. 63, no. 1, pp. 20-63, 1956.

[15] K. Yano and M. Kon, Structures on Manifolds, Series in Pure Mathematics, Worlds Scientific Publishing Co., Singapore, 1984.

[16] C. Gherghe, "Harmonicity on nearly trans-Sasaki manifolds," Demonstratio Mathematica, vol. 33, no. 1, pp. 151-157, 2000.

[17] D. E. Blair, D. K. Showers, and K. Yano, "Nearly Sasakian structure," Kodai Mathematical Seminar Reports, vol. 27, pp. 175-180, 1976.

[18] M. M. Tripathi and S. S. Shukla, "Semi-invariant submanifolds of nearly Kenmotsu manifolds," Bulletin of the Calcutta Mathematical Society, vol. 95, pp. 17-30, 2003.

[19] D. E. Blair, "Almost contact manifolds with killing structure tensors," Pacific Journal of Mathematics, vol. 39, no. 2, pp. 285-292, 1971.

[20] A. Lotta, "Slant submanifolds in contact geometry," Bulletin of Mathematical Society, Romania, vol. 39, pp. 183-198, 1996.

[21] B.-Y. Chen, "Cohomology of CR-submanifolds," Annales de la Faculté des sciences de Toulouse: Mathématiques, vol. 3, pp. 167-172, 1981.

[22] R. L. Bishop and B. O’Neill, "Manifolds of negative curvature," Transactions of the American Mathematical Society, vol. 145, pp. 1-49, 1969.

[23] B. Unal, Doubly warped products, PhD Thesis, University of Missouri-Columbia, 2000.

[24] B. Unal, "Doubly warped products," Differential Geometry and its Applications, vol. 15, no. 3, pp. 253-263, 2001. 